\title{
SB203580 protects against inflammatory response and lung injury in a mouse model of lipopolysaccharide-induced acute lung injury
}

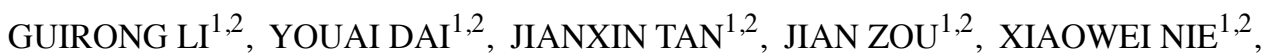 \\ ZHENKUN YANG ${ }^{1,2}$, JINGJING ZHAO $^{1,2}$, XUSHENG YANG ${ }^{1,2}$ and JINGYU CHEN ${ }^{1,3,4}$ \\ ${ }^{1}$ Center of Clinical Research; Departments of ${ }^{2}$ Neurosurgery and ${ }^{3}$ Cardiothoracic Surgery; ${ }^{4}$ Lung Transplant Group, \\ Wuxi People's Hospital of Nanjing Medical University, Wuxi, Jiangsu 214023, P.R. China
}

Received September 18, 2019; Accepted May 11, 2020

DOI: $10.3892 / \mathrm{mmr} .2020 .11214$

\begin{abstract}
Acute lung injury (ALI) is characterized by acute hypoxic respiratory failure, pulmonary edema and inflammatory infiltration. ALI has a high mortality rate $(\sim 30 \%)$ in the clinical setting; therefore, focusing on the treatment of lung edema and inflammatory responses in ALI is of significance. The present study investigated the effect of the p38 mitogen-activated protein kinase (p38MAPK) inhibitor, SB203580, on lung edema and inflammatory responses in ALI in vivo. A mouse model of ALI was established to assess the effect of SB203580 on edema, proinflammatory cytokine production, and the expression of interferon regulatory factor 5 (IRF5) and inducible nitric oxide synthase (iNOS) in lung tissues using immunoblotting, immunohistochemistry, immunofluorescence, hematoxylin and eosin staining, and ELISA. SB203580 inhibited LPS-induced lung injury and proinflammatory cytokine expression, including tumor necrosis factor- $\alpha$ and interleukin-1 $1 \beta$. SB203580 also downregulated LPS-induced IRF5 and iNOS expression, which are widely used as markers of proinflammatory macrophages. Collectively, the present study demonstrated that SB203580 protected against inflammatory responses and lung injury by inhibiting lung edema and downregulating proinflammatory mediators in LPS-induced lung injury.
\end{abstract}

\section{Introduction}

Acute lung injury (ALI) is a clinical syndrome characterized by acute hypoxemic respiratory failure, pulmonary infiltration and edema in the lung tissues, of which the most severe

Correspondence to: Professor Jingyu Chen, Center of Clinical Research, Wuxi People's Hospital of Nanjing Medical University, 299 Qingyang Road, Wuxi, Jiangsu 214023, P.R. China

E-mail: cjy_wx@163.com

Key words: acute lung injury, SB203580, inflammatory response, interferon regulatory factor 5 manifestation is acute respiratory distress syndrome (1). The mortality rate of patients with ALI is $\sim 30 \%$, which has not changed over the past several years $(2,3)$. The increased permeability of the alveolocapillary membrane is an important physiological alteration that is observed in patients with ALI, which is induced by alveolocapillary barrier dysfunction and epithelial injury. Increased permeability of the alveolocapillary membrane causes lung edema due to infiltration of large amounts of intravascular protein-rich fluid into the pulmonary interstitial and alveolar space (4). Moreover, overwhelming inflammation is also a critical pathophysiological process observed in patients with ALI (5). Previous studies suggested that the extensive infiltration of neutrophils and the presence of resident and recruited macrophages could mediate robust inflammatory responses in patients with ALI and animal models of ALI (6,7). Immune cells that accumulate in lung tissues release various proinflammatory mediators, such as interleukin (IL)-6, tumor necrosis factor (TNF)- $\alpha$ and interferon (IFN) $-\gamma$, which exacerbate inflammation. Moreover, immune cells, including proinflammatory macrophages, produce nitric oxide (NO) $(8,9)$.

Interferon regulatory factor 5 (IRF5) is widely expressed in immune cells, including macrophages, B-cells, monocytes and dendritic cells (10-12). Previous studies demonstrated that IRF5 mediated the activation of inflammatory macrophages in vivo and classical macrophages in vitro (10-12). Activation of proinflammatory macrophages leads to excessive secretion of TNF- $\alpha$, IL-6, IL-12 and IL-23, and downregulation of IL-10 and transforming growth factor- $\beta$ (13). Therefore, the expression of IRF5 may also promote inflammatory macrophage activation and inflammatory responses in lung tissue.

It has been reported that p38 mitogen-activated protein kinase (p38MAPK) serves a crucial role in intracellular inflammatory signaling pathways, including the TNF and TLR4 signaling pathways (14). However, the effects of SB203580, an inhibitor of p38MAPK, on lung tissue injury and the inflammatory response are not completely understood. In the present study, a mouse model of ALI was established by 24-h lipopolysaccharide (LPS) stimulation. The aim of the present study was to investigate the effect of SB203580 on inflammatory response and lung injury in ALI. 


\section{Materials and methods}

Animals and treatment. In the present study, a total of 45 healthy male C57BL/6 mice (age, 8 weeks; weight, 20-25 g) were purchased from Changzhou Cavans Experimental Animal Co., Ltd. The animals were kept under standardized conditions with a mean room temperature of $22-24^{\circ} \mathrm{C}$, a $12 / 12 \mathrm{~h}$ light/dark cycle and a humidity of 50-60\%. The mice were fed a standard animal diet with food and tap water ad libitum. The mice were randomly divided into three groups (Ctrl, ALI and SB203580 groups), each containing 15 mice. All experiments were approved by The Institutional Animal Care and Use Committee at Nanjing Medical University.

LPS causes ALI after intratracheal administration and reduces associated non-pulmonary organ dysfunction, whereas intravenous administration does not lead to tissue-specific lung injury $(15,16)$. Therefore, in the present study, mice were treated with $50 \mu 1(5 \mathrm{mg} / \mathrm{kg})$ LPS (Escherichia coli O111:B4; cat. no. L2630; Sigma-Aldrich; Merck KGaA) by intratracheal injection under anesthesia with $40 \mathrm{mg} / \mathrm{kg}$ pentobarbital sodium by intraperitoneal injection. In the control (Ctrl) group, mice were treated with an equal volume of PBS by intratracheal injection. The absorption rate of intraperitoneal injection is lower compared with intratracheal injection, therefore, SB203580 injection was performed prior to LPS treatment. Thus, in the SB203580 group, mice received $20 \mathrm{mg} / \mathrm{kg} \mathrm{SB} 203580$ (cat. no. S1863; Beyotime Institute of Biotechnology) by intraperitoneal injection $1 \mathrm{~h}$ before LPS stimulation. A total of 30 mice were anesthetized by isoflurane inhalation (5\%) and then sacrificed by cervical dislocation at $24 \mathrm{~h}$ post-LPS treatment. For the histological examination, immunohistochemistry and immunofluorescence assays, a further 15 mice were anesthetized by the intraperitoneal injection of pentobarbital sodium $(40 \mathrm{mg} / \mathrm{kg}$ ) and sacrificed by myocardial perfusion fixation after LPS stimulation for $24 \mathrm{~h}$. Bronchoalveolar lavage fluid (BALF) and lung tissues were collected for subsequent analysis at $24 \mathrm{~h}$ post-LPS treatment, since the features of ALI in the model are close to the diagnostic criteria of patients with ALI at this time point $(15,17)$.

Histological examination of lung tissue. At $24 \mathrm{~h}$ post-LPS treatment, tissue from the middle lobe of the right lung was collected, fixed with $10 \%$ formaldehyde solution at room temperature for $24 \mathrm{~h}$, dehydrated using a graded ethanol series, embedded in paraffin and cut into $4-\mu \mathrm{m}$ sections. The sections were stained with hematoxylin for $10 \mathrm{~min}$, followed by eosin for $3 \mathrm{~min}$, at room temperature. Sections were observed under a BX53 light microscope (magnification, x400; Olympus Corporation). Lung tissue injuries were scored according to a previous study (18).

Evaluation of pulmonary edema. Native tissue from the right lower lobe of the lung was weighed to obtain the 'wet weight' (W). Subsequently, to obtain the 'dry weight' (D), the tissues was dried at $65^{\circ} \mathrm{C}$ for $72 \mathrm{~h}$ and then weighed. Pulmonary edema was evaluated by calculating the W/D ratio.

$B A L F$ collection and cell counting. The pulmonary lavage was performed three times with $1 \mathrm{ml}$ PBS. The collected BALF was centrifuged at $845 \mathrm{xg}$ for $10 \mathrm{~min}$ at $4^{\circ} \mathrm{C}$. Subsequently, the cell pellet was resuspended with $1 \mathrm{ml}$ PBS. The total number of BALF cells was counted using a hematocytometer under a light microscope (magnification, x200).

Immunohistochemistry. To assess the expression and distribution of IRF5 in lung tissue, the middle lobe of the right lung was fixed in $10 \%$ formaldehyde at room temperature for $24 \mathrm{~h}$, then dehydrated and embedded in paraffin. All samples in paraffin were cut into $4-\mu \mathrm{m}$ sections. Subsequently, 4- $\mu \mathrm{m}$ sections were dewaxed and rehydrated through graded alcohol at room temperature and then subjected to antigen retrieval by high pressure in $10 \mathrm{mmol} / \mathrm{l}$ citrate acid $(\mathrm{pH} 6.0)$ for $8 \mathrm{~min}$. The sections were blocked with $10 \%$ goat serum (cat. no. AR0009; Boster Biological Technology) at room temperature for $1 \mathrm{~h}$. The sections were incubated with an IRF5 primary antibody (cat. no. ab181553; 1:600; Abcam) at $4^{\circ} \mathrm{C}$ overnight. The sections were washed using PBS and then were incubated with a goat anti-rabbit biotinylated secondary antibody (cat. no. ab6720; 1:1,000; Abcam) at room temperature for $1.5 \mathrm{~h}$, and streptavidin-horseradish peroxidase (HRP; 1:10,000; cat. no. ab7403; Abcam) at room temperature for $45 \mathrm{~min}$, respectively. Chromagen detection was performed with the DAB substrate kit (cat. no. ab64238; Abcam), according to the manufacturer's instructions. Lung tissue sections were counterstained with hematoxylin for $8 \mathrm{~min}$ at room temperature and rinsed with distilled water, dehydrated through gradient ethanol and xylene, then mounted with mounting medium (cat. no. 14177; Cell Signaling Technology, Inc.). Images were captured using a BX53 light microscope (magnification, x400; Olympus Corporation). Quantification was performed by calculating the percentage of IRF5-positive cells. IRF5-positive cells and total cells were counted in five sections and five different fields/section.

Western blot analysis of the lung tissues. Native tissue from the left lobe of the lung was homogenized and protein samples were extracted using RIPA buffer (cat. no. P0013B; Beyotime Institute of Biotechnology). Protein content was determined using BCA kit (cat. no. P0012S; Beyotime Institute of Biotechnology). Proteins ( $30 \mu \mathrm{g} / \mathrm{line}$ ) were separated via SDS-PAGE on $10 \%$ gel and transferred to PVDF membranes. PVDF membranes were blocked using $5 \%$ non-fat milk at room temperature for $1 \mathrm{~h}$ and then incubated with primary antibodies targeted against IRF5 (cat. no. ab181553; 1:1,000; Abcam), inducible NO synthase (iNOS; cat. no. ab15323; 1:1,000; Abcam), arginase 1 (Arg1; cat. no. 93668; 1:1,000; Cell Signaling Technology, Inc.) and $\beta$-actin (cat. no. ab8227; 1:2,000; Abcam) at $4^{\circ} \mathrm{C}$ overnight. Following primary incubation, membranes were incubated with HRP-conjugated goat anti-rabbit IgG (cat. no. A0208; 1:1000; Beyotime Institute of Biotechnology) secondary antibodies at room temperature for $1.5 \mathrm{~h}$. In addition, enhanced chemiluminescence reagents (cat. no. WBKLS0100; EMD Millipore) were used for imaging and quantification was performed using Image J software version 1.47 (National Institutes of Health) with $\beta$-actin as the loading control.

Immunofluorescence. Following treatment with LPS for $24 \mathrm{~h}$, mice were subjected to myocardial perfusion fixation to preserve tissue. Tissue from the left lung was fixed with 
A

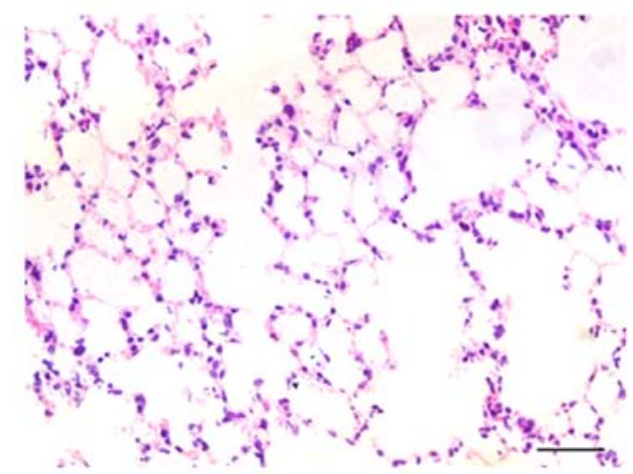

C

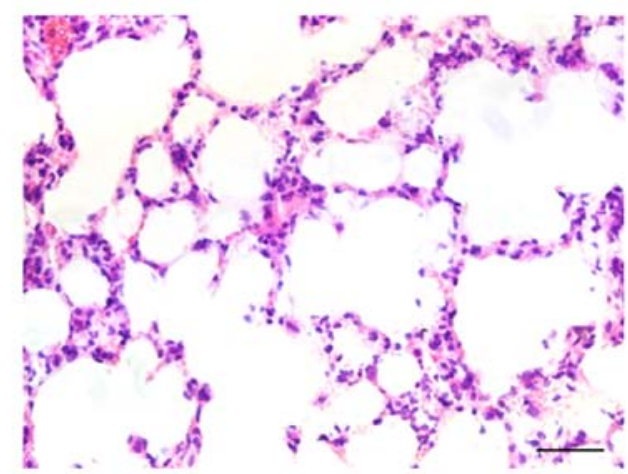

$\mathrm{B}$

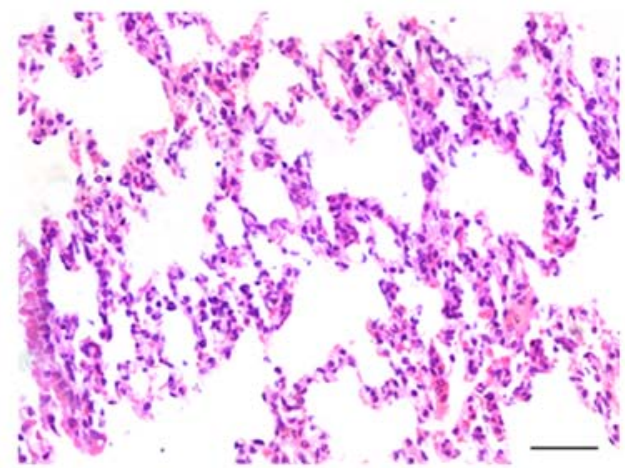

D

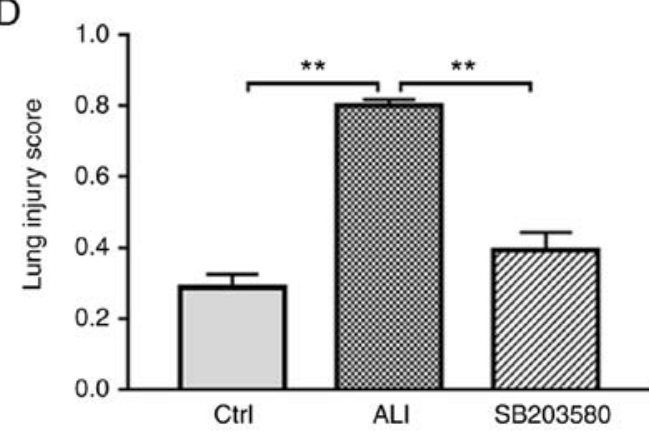

Figure 1. Histopathological alterations in lung tissues. C57BL/6 mice were stimulated with lipopolysaccharide or PBS, and treated with SB203580. Lung sections from the (A) Ctrl, (B) ALI and (C) SB203580 groups were stained with hematoxylin and eosin and visualized by light microscopy (magnification, x400; scale bar, $50 \mu \mathrm{m}$ ). (D) Lung injury scores. ${ }^{* *} \mathrm{P}<0.01$, as indicated. Ctrl, control; ALI, acute lung injury.

$10 \%$ formaldehyde solution at room temperature for $4 \mathrm{~h}$, dehydrated in $30 \%$ sucrose, embedded in optimal cutting temperature reagent and cut into $7-\mu \mathrm{m}$ sections using a freezing microtome. The sections were blocked with $10 \%$ goat serum (cat. no. AR0009; Boster Biological Technology) at room temperature for $40 \mathrm{~min}$. The frozen lung sections were stained with F4/80 (1:50; cat. no. ab16911; Abcam) and iNOS (1:50; cat. no. ab15323; Abcam) primary antibodies at $4^{\circ} \mathrm{C}$ overnight. Subsequently, the sections were incubated with anti-rat Alexa Fluor ${ }^{\circledR}$ 594-conjugated (1:500; cat. no. A-11007; Invitrogen; Thermo Fisher Scientific, Inc.) and anti-rabbit Alexa Fluor ${ }^{\circledR}$ 488-conjugated (1:500; cat. no. A-11034; Invitrogen; Thermo Fisher Scientific, Inc.) secondary antibodies at room temperature for $2 \mathrm{~h}$. The localization of protein was examined by confocal laser scanning microscopy (magnification, x200; Leica Microsystems $\mathrm{GmbH}$ ).

Measurements of cytokines. Following LPS treatment for $24 \mathrm{~h}$, lung tissues were ground into protein homogenate. The levels of the proinflammatory cytokines TNF- $\alpha$ (cat. no. MTA00B) and IL-1 $\beta$ (cat. no. MLB00C) were measured using ELISA kits (both R\&D Systems, Inc.) according to the manufacturer's instructions.

Statistical analysis. All experiments were repeated at least three times and the data are presented as the mean \pm SEM. Statistical analyses were performed using GraphPad Prism software (version 7; GraphPad Software, Inc.). Multiple comparisons were analyzed using one-way ANOVA followed by Tukey's post hoc test. $\mathrm{P}<0.05$ was considered to indicate a statistically significant difference.

\section{Results}

SB203580 attenuates LPS-induced lung histopathological alterations. Histopathological alterations in the lung were detected using hematoxylin and eosin (H\&E) staining (Fig. 1). Lung sections of Ctrl group showed a normal alveolar morphology (Fig. 1A). Histological sections from the lungs of LPS-induced mice displayed edema, as well as basal membrane and alveolar wall thickening (Fig. 1B). By contrast, treatment with SB203580 markedly attenuated LPS-induced lung injury (Fig. 1C). The lung injury score in the ALI group was significantly increased compared with the Ctrl group. However, treatment with SB203580 significantly decreased the lung injury score compared with the ALI group (Fig. 1D). Thus, the intraperitoneal injection of SB203580 significantly decreased lung injury in ALI model mice.

SB203580 reduces LPS-induced lung edema and inflammatory responses in vivo. Lung tissue edema was assessed by calculating the W/D weight ratio. The lung W/D ratio was significantly decreased in the SB203580 group compared with the ALI group (Fig. 2A). In addition, inflammation was evaluated by calculating the total number of cells in the BALF and measuring proinflammatory cytokine levels in lung tissues. Total cell counts in the BALF were higher in the ALI group compared with the Ctrl group. However, treatment with SB203580 significantly decreased cell counts in total BALF compared with the ALI group (Fig. 2B). Similarly, the levels of proinflammatory cytokines TNF- $\alpha$ and IL- $1 \beta$ were significantly elevated in the ALI group compared with 
A

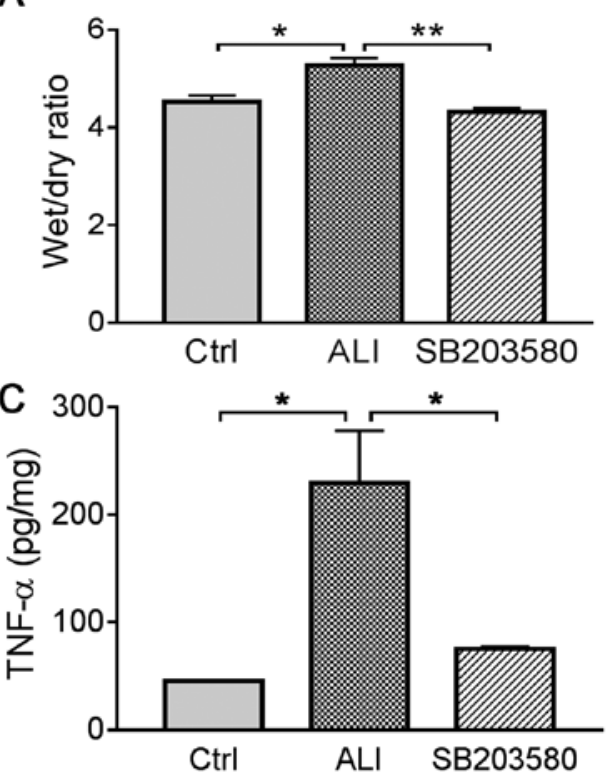

B

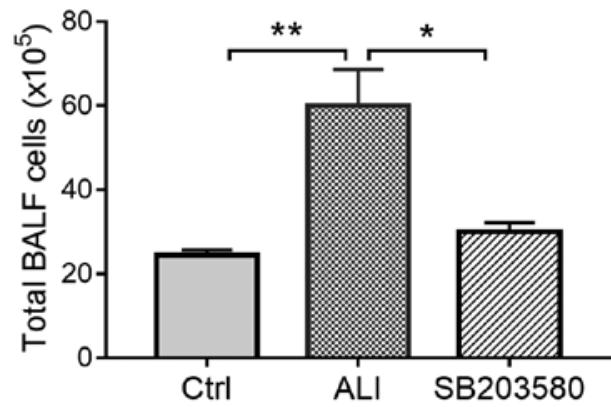

D

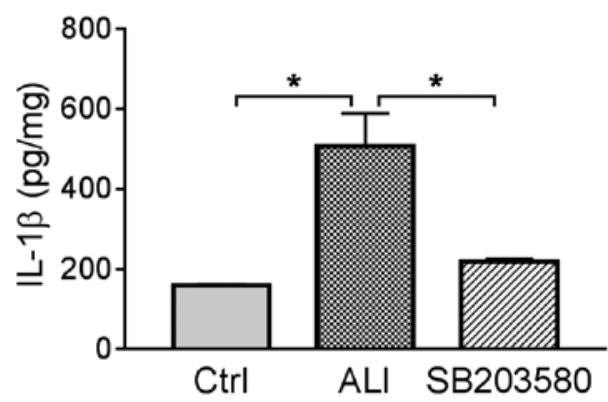

Figure 2. SB203580 suppresses lipopolysaccharide-induced lung edema and inflammatory responses. (A) Lung tissue wet/dry weight ratio. (B) Total BALF cell numbers. Lung tissue was collected and ground into protein homogenate for the measurement of proinflammatory (C) TNF- $\alpha$ and (D) IL-1 $\beta$ cytokine levels. $\mathrm{n}=5$. ${ }^{*} \mathrm{P}<0.05$ and ${ }^{* *} \mathrm{P}<0.01$, as indicated. BALF, bronchoalveolar lavage fluid; TNF- $\alpha$, tumor necrosis factor- $\alpha$; IL, interleukin; Ctrl, control; ALI, acute lung injury.

A
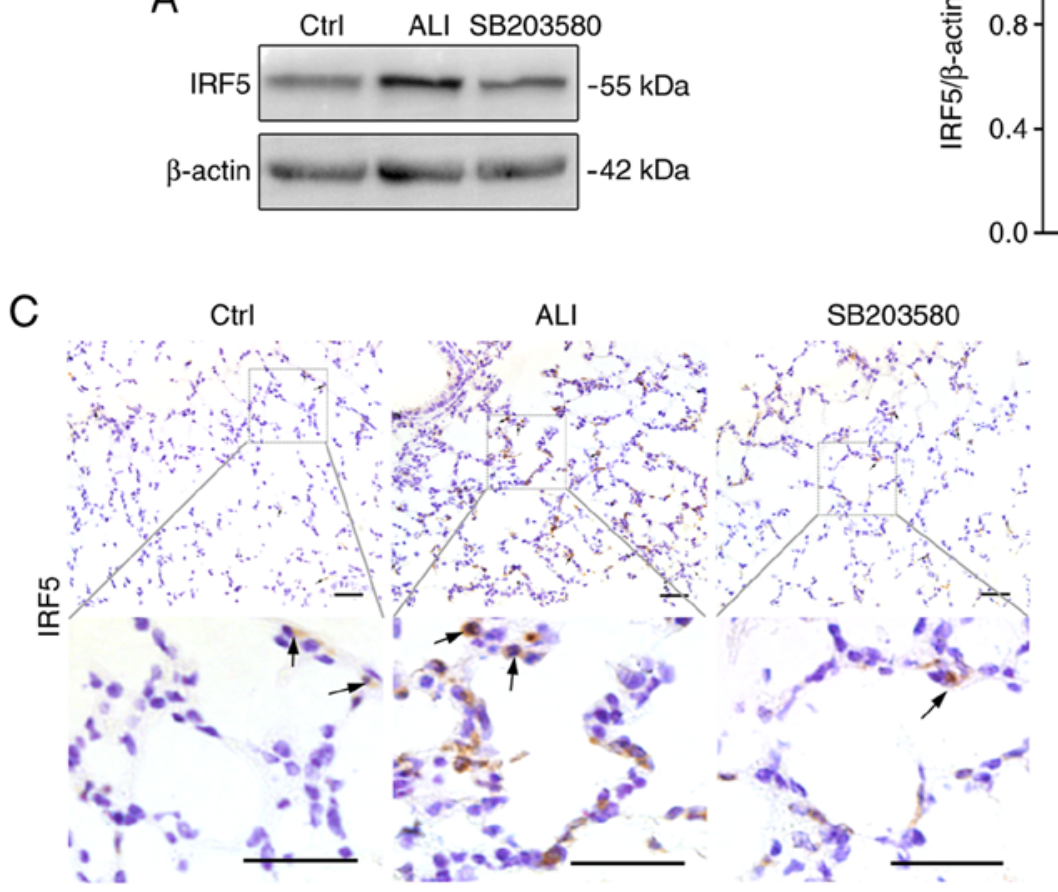

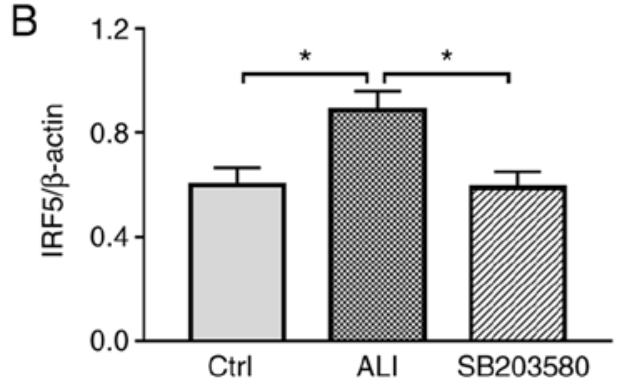

D

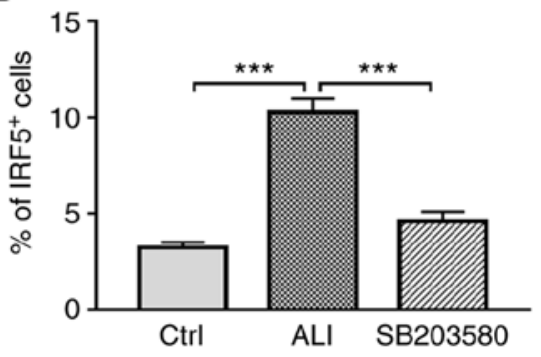

Figure 3. SB203580 reverses LPS-induced IRF5 expression in lung tissue. Pulmonary expression of IRF5 was (A) determined by western blotting and (B) semi-quantified. (C) Effect of SB203580 on the expression and distribution of IRF5 in lung tissues (scale bar, $40 \mu \mathrm{m}$ ). (D) IRF5 expression was quantified by calculating the percentage of positive cells (brown) relative to total cells [brown + blue (hematoxylin)] in lung tissue sections. IRF5-positive cells and total cells were counted in five sections and five different fields/section. $\mathrm{n}=5 .{ }^{*} \mathrm{P}<0.05$ and ${ }^{* * *} \mathrm{P}<0.001$, as indicated. ALI, acute lung injury; IRF5, interferon regulatory factor 5; Ctrl, control.

the Ctrl group, and treatment with SB203580 significantly decreased the levels of TNF- $\alpha$ and IL-1 $\beta$ compared with the ALI group (Fig. 2C and D).
SB203580 inhibits IRF5 expression in the lung tissues of ALI model mice. The effect of SB203580 on IRF5 expression was assessed by western blotting and immunohistochemical 

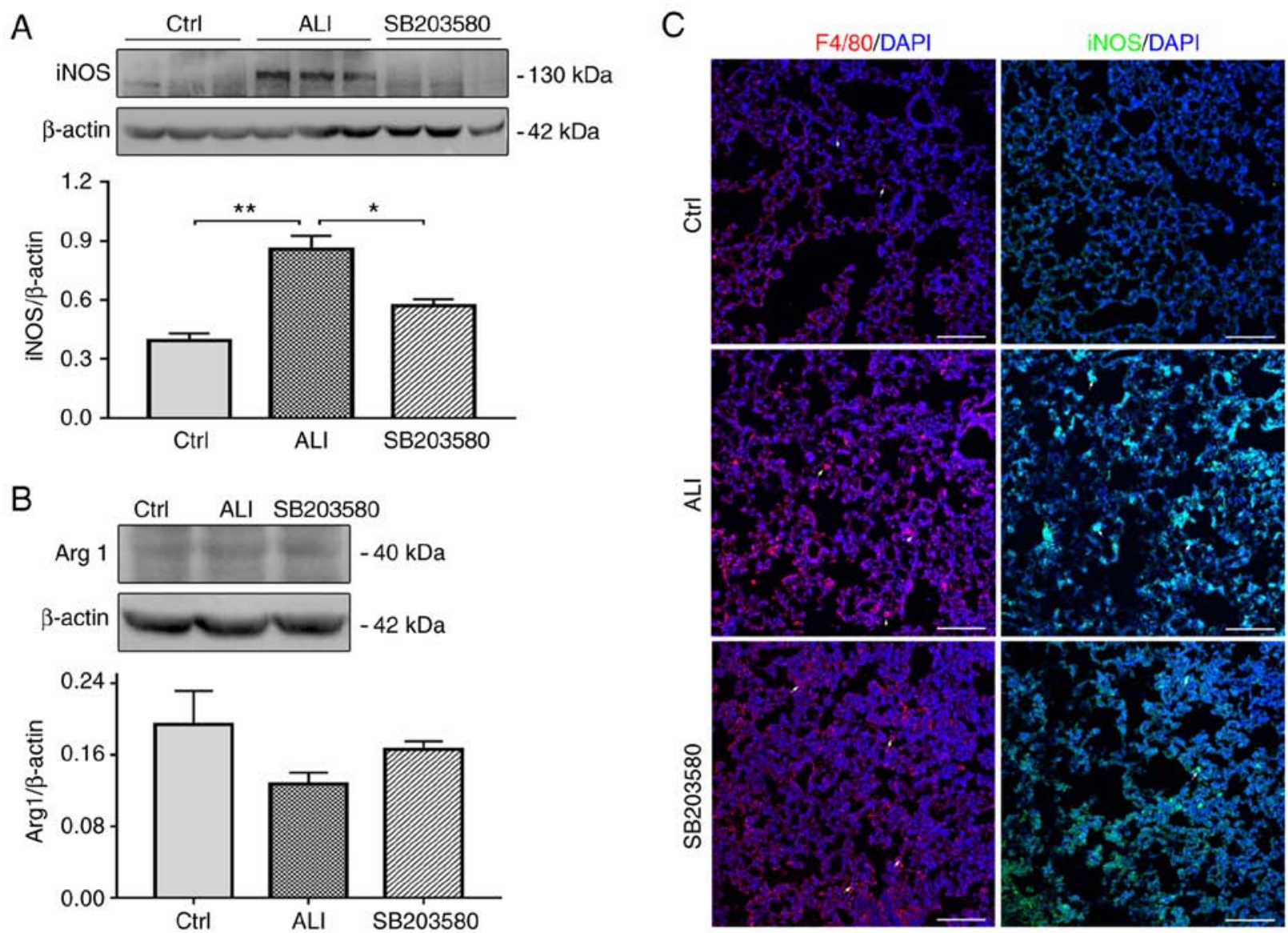

Figure 4. SB203580 decreases the expression of iNOS and F4/80 in lung tissues. (A) SB203580 downregulated LPS-induced iNOS expression. (B) SB203580 had no effect on the expression of Arg1. (C) Frozen lung tissue sections were stained with antibodies targeted against F4/80 (left) and iNOS (right), and imaged by confocal microscopy (magnification, $\mathrm{x} 200$; scale bar, $100 \mu \mathrm{m}$ ). $\mathrm{n}=5 .{ }^{*} \mathrm{P}<0.05$ and ${ }^{* *} \mathrm{P}<0.01$, as indicated. iNOS, inducible nitric oxide synthase; ALI, acute lung injury; Arg1, arginase 1; Ctrl, control.

staining. The levels of IRF5 were significantly increased in ALI model mice compared with Ctrl mice. Treatment with SB203580 resulted in a significant decrease in IRF5 expression levels compared with the ALI group (Fig. 3A and B). IRF5 expression was further investigated by immunohistochemical staining (Fig. 3C). The frequency of IRF5-postive cells was significantly increased in the lungs in the ALI group compared with the Ctrl group; however, treatment with SB203580 significantly inhibited LPS-induced IRF5 expression (Fig. 3D).

SB203580 decreases the expression of iNOS and F4/80 in the lung tissues of ALI model mice. iNOS is an inflammatory gene marker, which serves a critical role in LPS-induced ALI (19). In the present study, the effect of SB203580 on iNOS expression was evaluated using western blotting and immunofluorescence assays. The expression of iNOS was significantly increased in the lung tissues of ALI model mice compared with Ctrl mice. However, LPS-induced iNOS expression was significantly decreased following SB203580 treatment (Fig. 4A and C). The levels of Arg1, were low in the Ctrl group and remained unchanged in the ALI group (Fig. 4B).

$\mathrm{F} 4 / 80$ is widely used as a macrophage marker, although it is also expressed on monocytes and neutrophils in mice $(20,21)$. The expression of F4/80 was increased in ALI lung tissues compared with Ctrl lung tissues, which was inhibited by SB203580 treatment (Fig. 4C).

\section{Discussion}

ALI is a common clinical condition characterized by hypoxia, edema and inflammatory cell infiltration of neutrophils and macrophages into lung tissues, leading to respiratory failure $(22,23)$. Alveolar epithelium and capillary endothelium injuries increase alveolar barrier permeability, resulting in edema, inflammatory leukocyte infiltration and bleeding $(4,24)$. Focusing on the treatment of lung edema and inflammatory injury in patients with ALI is of significance. Mouse models of LPS-induced lung injury have been widely used to study the pathogenesis of ALI $(4,18)$. The mild LPS-induced mouse model of ALI used in the present study displayed edema and inflammation in lung tissues.

Inhibition of proinflammatory cytokine production allows the regulation of inflammatory responses (25). The p38MAPK signaling pathway has a key regulatory role in inflammatory processes taking place in the lung (26). M39, an inhibitor of p38MAPK, inhibits the activation of p38MAPK in neutrophils and macrophages in vitro, which prevents the release of TNF- $\alpha$ and macrophage inflammatory protein 2 , limits the activation of p38MAPK in vivo and reduces the accumulation of neutrophils in the airway. Moreover, the novel synthesized flavonoid 
LFG-500 can alleviate LPS-induced inflammatory responses by inhibiting the expression of IL-6, TNF- $\alpha$ and IL-1 $\beta$ (27).

In the present study, the effects of SB203580, an inhibitor of p38MAPK, on ALI and inflammation in LPS-induced mice were investigated. SB203580 treatment significantly reduced LPS-induced expression of TNF- $\alpha$ and IL-1 $\beta$ in lung tissues, which suggested that SB203580 inhibited ALI-stimulated inflammatory responses by inhibiting the expression of proinflammatory cytokines. Furthermore, SB203580 reduced the W/D weight ratio compared with the ALI group, indicating attenuation of LPS-induced lung edema.

The transcription factor IRF5 is involved in multiple autoimmune diseases, including systemic lupus erythematosus, rheumatoid arthritis and inflammatory bowel disease (28). IRF5 induces the upregulation of proinflammatory genes, repression of anti-inflammatory mediators and polarization of macrophages to a proinflammatory phenotype (28-30). In the present study, the expression of IRF5 was significantly upregulated in ALI model mice compared with Ctrl mice, and significantly downregulated in SB203580-treated ALI model mice. The results suggested that SB203580 inhibited LPS-stimulated inflammatory responses by decreasing the expression of IRF5 in lung tissue.

iNOS is an enzyme expressed in macrophages and endothelial cells that synthesizes NO via L-arginase oxidation. iNOS competes with Arg1 for the same substrate and is considered a marker of inflammatory responses $(31,32)$. In the present study, the expression of iNOS was increased in the lungs of ALI mice compared with Ctrl mice, and SB203580 treatment decreased LPS-induced iNOS expression. By contrast, the expression of Arg1 in lung tissues remained almost unchanged among the three groups. Thus, the results suggested that SB203580 attenuated the inflammatory response by decreasing the expression of iNOS in LPS-induced lung tissues.

In conclusion, the inhibitor of the p38MAPK signaling pathway SB203580 attenuated lung injury and inflammatory responses in ALI model mice. Treatment with SB203580 in LPS-induced ALI model mice reduced edema and the expression of proinflammatory cytokines, and alleviated pathological changes in lung tissues. SB203580 also decreased the expression of IRF5 and iNOS in ALI lung tissues. Thus, IRF5 may serve as a key regulatory factor of the inflammatory response in the lungs and may exert its effects by promoting a proinflammatory macrophage phenotype. Although further research is required to fully understand the mechanism of SB203580 attenuating lung edema and inflammatory response in ALI, the present study suggested a protective role of SB203580 in LPS-induced ALI; therefore, SB203580 may serve as a potential preventive agent for ALI.

\section{Acknowledgements}

Not applicable.

\section{Funding}

The present study was supported by the Natural Science Foundation of Jiangsu Province (grants nos. BK20190150 and BK20160196) and the National Natural Science Foundation of China (grant no. 81500039).

\section{Availability of data and materials}

The datasets used and/or analyzed during the present study are available from the corresponding author upon reasonable request.

\section{Authors' contributions}

GL designed the study, analyzed the data and wrote the manuscript. YD performed the hematoxylin and eosin staining and immunohistochemistry assay. JT performed immunofluorescence and ELISA assays. JZo and XN analyzed the data and helped to write the paper. ZY, JZh and XY performed animal experiments and western blot assays. JC designed the present study and provided financial support for this work. All authors read and approved the final manuscript.

\section{Ethics approval and consent to participate}

All experiments in the present study were approved by The Institutional Animal Care and Use Committee at Nanjing Medical University.

\section{Patient consent for publication}

Not applicable.

\section{Competing interests}

The authors declare that they have no competing interests.

\section{References}

1. Ashbaugh DG, Bigelow DB, Petty TL and Levine BE: Acute respiratory distress in adults. Lancet 2: 319-323, 1967.

2. Villar J, Blanco J and Kacmarek RM: Current incidence and outcome of the acute respiratory distress syndrome. Curr Opin Crit Care 22: 1-6, 2016.

3. Erickson SE, Martin GS, Davis JL, Matthay MA and Eisner MD; NIH NHLBI ARDS Network: Recent trends in acute lung injury mortality: 1996-2005. Crit Care Med 37: 1574-1579, 2009.

4. Patel BV, Wilson MR and Takata M: Resolution of acute lung injury and inflammation: A translational mouse model. Eur Respir J 39: 1162-1170, 2012.

5. Gill SE, Yamashita CM and Veldhuizen RA: Lung remodeling associated with recovery from acute lung injury. Cell Tissue Res 367: 495-509, 2017.

6. Matute-Bello G, Frevert CW and Martin TR: Animal models of acute lung injury. Am J Physiol Lung Cell Mol Physiol 295: L379-L399, 2008.

7. Han S and Mallampalli RK: The acute respiratory distress syndrome: From mechanism to translation. J Immunol 194: 855-860, 2015.

8. Robb CT, Regan KH, Dorward DA and Rossi AG: Key mechanisms governing resolution of lung inflammation. Semin Immunopathol 38: 425-448, 2016.

9. Freire MO and Van Dyke TE: Natural resolution of inflammation. Periodontol 2000 63: 149-164, 2013.

10. Krausgruber T, Blazek K, Smallie T, Alzabin S, Lockstone H, Sahgal N, Hussell T, Feldmann M and Udalova IA: IRF5 promotes inflammatory macrophage polarization and TH1-TH17 responses. Nat Immunol 12: 231-238, 2011.

11. Lien C, Fang CM, Huso D, Livak F, Lu R and Pitha PM: Critical role of IRF-5 in regulation of B-cell differentiation. Proc Natl Acad Sci USA 107: 4664-4668, 2010.

12. Krausgruber T, Saliba D, Ryzhakov G, Lanfrancotti A, Blazek K and Udalova IA: IRF5 is required for late-phase TNF secretion by human dendritic cells. Blood 115: 4421-4430, 2010. 
13. Aggarwal NR, King LS and D'Alessio FR: Diverse macrophage populations mediate acute lung inflammation and resolution. Am J Physiol Lung Cell Mol Physiol 306: L709-L725, 2014.

14. Schnyder-Candrian S, Quesniaux VF, Di Padova F, Maillet I, Noulin N,CouillinI,MoserR,ErardF, VargaftigBB, Ryffel B, et al: Dual effects of p38 MAPK on TNF-dependent bronchoconstriction and TNF-independent neutrophil recruitment in lipopolysaccharide-induced acute respiratory distress syndrome. J Immunol 175: 262-269, 2005.

15. Szarka RJ, Wang N, Gordon L, Nation PN and Smith RH: A murine model of pulmonary damage induced by lipopolysaccharide via intranasal instillation. J Immunol Methods 202: 49-57, 1997.

16. Chen H, Bai $\mathrm{C}$ and Wang $\mathrm{X}$ : The value of the lipopolysaccharide-induced acute lung injury model in respiratory medicine. Expert Rev Respir Med 4: 773-783, 2010.

17. van Helden HP, Kuijpers WC, Steenvoorden D, Go C, Bruijnzeel PL, van Eijk M and Haagsman HP: Intratracheal aerosolization of endotoxin (LPS) in the rat: A comprehensive animal model to study adult (acute) respiratory distress syndrome. Exp Lung Res 23: 297-316, 1997.

18. Matute-Bello G, Downey G, Moore BB, Groshong SD, Matthay MA, Slutsky AS and Kuebler WM; Acute Lung Injury in Animals Study Group: An official American Thoracic Society workshop report: Features and measurements of experimental acute lung injury in animals. Am J Respir Cell Mol Biol 44: 725-738, 2011 .

19. Mehta S: The effects of nitric oxide in acute lung injury. Vascul Pharmacol 43: 390-403, 2005.

20. Taylor PR, Martinez-Pomares L, Stacey M, Lin HH, Brown GD and Gordon S: Macrophage receptors and immune recognition. Annu Rev Immunol 23: 901-944, 2005.

21. Gordon S and Mantovani A: Diversity and plasticity of mononuclear phagocytes. Eur J Immunol 41: 2470-2472, 2011.

22. Hughes KT and Beasley MB: Pulmonary manifestations of acute lung injury: More than just diffuse alveolar damage. Arch Pathol Lab Med 141: 916-922, 2017.
23. Butt Y, Kurdowska A and Allen TC: Acute Lung Injury: A Clinical and Molecular Review. Arch Pathol Lab Med 140: 345-350, 2016.

24. Elicker BM, Jones KT, Naeger DM and Frank JA: Imaging of Acute Lung Injury. Radiol Clin North Am 54: 1119-1132, 2016.

25. Pyee Y, Chung HJ, Choi TJ, Park HJ, Hong JY, Kim JS, Kang SS and Lee SK: Suppression of inflammatory responses by handelin, a guaianolide dimer from Chrysanthemum boreale, via downregulation of NF- $\kappa \mathrm{B}$ signaling and pro-inflammatory cytokine production. J Nat Prod 77: 917-924, 2014.

26. Nick JA, Young SK, Brown KK, Avdi NJ, Arndt PG, Suratt BT, Janes MS, Henson PM and Worthen GS: Role of p38 mitogen-activated protein kinase in a murine model of pulmonary inflammation. J Immunol 164: 2151-2159, 2000.

27. Li C, Yang D, Cao X, Wang F, Jiang H, Guo H, Du L, Guo Q and Yin X: LFG-500, a newly synthesized flavonoid, attenuates lipopolysaccharide-induced acute lung injury and inflammation in mice. Biochem Pharmacol 113: 57-69, 2016.

28. Eames HL, Corbin AL and Udalova IA: Interferon regulatory factor 5 in human autoimmunity and murine models of autoimmune disease. Transl Res 167: 167-182, 2016.

29. Khoyratty TE and Udalova IA: Diverse mechanisms of IRF5 action in inflammatory responses. Int J Biochem Cell Biol 99: 38-42, 2018.

30. Weiss M, Blazek K, Byrne AJ, Perocheau DP and Udalova IA: IRF5 is a specific marker of inflammatory macrophages in vivo. Mediators Inflamm 2013: 245804, 2013.

31. Cook HT, Jansen A, Lewis S, Largen P, O'Donnell M, Reaveley D and Cattell V: Arginine metabolism in experimental glomerulonephritis: Interaction between nitric oxide synthase and arginase. Am J Physiol 267: F646-F653, 1994.

32. Li Z, Zhao ZJ, Zhu XQ, Ren QS, Nie FF, Gao JM, Gao XJ, Yang TB, Zhou WL, Shen JL, et al: Differences in iNOS and arginase expression and activity in the macrophages of rats are responsible for the resistance against $\mathrm{T}$. gondii infection. PLoS One 7: e35834, 2012. 